\title{
Determinantes de la transmisión de dengue en Veracruz: un abordaje ecológico para su control
}

Javier Escobar-Mesa, Biol, MSP, ${ }^{(1)}$ Héctor Gómez-D antés, MC, M en C.. ${ }^{(2)}$

\begin{abstract}
Escobar-Mesa J, Gómez-Dantés H.
Determinantes de la transmisión de dengue en Veracruz:

un abordaje ecológico para su control.

Salud Publica Mex 2003;45:43-53.

El texto completo en inglés de este artículo está disponible en: http://www.insp.mx/salud/index.html
\end{abstract}

\section{Resumen}

Objetivo. Identificar los factores ambientales, sociales y demográficos que contribuyen a explicar las diferencias en la transmisión de dengue en Veracruz, México, durante el periodo 1995-1998 y definir las áreas de riesgo para promover un mejor control del problema. Material y métodos. Bajo un diseño ecoló gico se analizó una base de datos con el número de casos de dengue que se presentaron en 1249 localidades en las 11 Jurisdicciones Sanitarias del estado de Veracruz durante el perio do 1995-1998. Cada una de las localidades fue identificada de acuerdo con su altitud, latitud y longitud, y con el número total de la población por sexo, el número de viviendas y la dotación de servicios públicos. Se to mó también el índice de marginación por localidad del Consejo N acional de Población. Resultados. El dengue en Veracruz se registró en $17 \%$ de las localidades y en sólo $6 \%$ de ellas se concentraron $70 \%$ de los casos de dengue en el estado. Las localidades repetidoras de dengue fueron grandes centros urbanos, con baja marginación y extensa dotación de ser vicios públicos. El dengue sí se transmite en las áreas rurales del estado aunque la transmisión es ocasional. El promedio de casos de dengue reportados en las localidades dependió del tamaño de la misma y del número de años que reportaron dengue durante el periodo. Se reporta un umbral poblacional para la transmisión. Conclusiones. Se identificaron las localidades repetidoras de dengue que concentran $70 \%$ de los casos, y las determi-

\author{
Escobar-Mesa J, Gómez-Dantés H. \\ Dengue fever transmission in Veracruz, Mexico: \\ An ecological approach to disease control. \\ Salud Publica Mex 2003;45:43-53. \\ The English version of this paper \\ is available at: http://www.insp.mx/salud/index.html
}

\begin{abstract}
A bstract
Objective. To assess the ecological, social, and demographic factors associated with the transmission of dengue virus infection in Veracruz, Mexico, and to identify risk areas to target control measures. Material and Methods. This ecolo gical study included data for 1,249 localities within the 11 $H$ ealth Jurisdictions of the State ofVeracruz, M exico, for the 1995-1998 period. The following data were collected for each locality: total number of cases per year, population by sex, number of households, provision of public services, altitude, latitude, longitude, and deprivation index. Results. D engue transmission was registered in $17 \%$ of the localities in the State; $70 \%$ of the cases were concentrated in only $6 \%$ of the localities. Recurrent localities were urban centers with adequate availability of public services and low deprivation indices. D engue transmission was detected in rural areas, but it was not common. The average number of cases differed according to the size of the locality and the number of years dengue was reported. A population threshold to maintain transmission was found. Conclusion. Recurrent localities concentrating $70 \%$ of dengue fever cases were identified, as well as the ecological and demographic factors associated with dengue transmission. The risk stratification approach to dengue transmission may improve control and prevention of this disease in high-risk areas. The English version of this paper is available at: http://www.insp.mx/salud/ index.html
\end{abstract}

(1) Programa de Prevención y Control de Enfermedades Transmitidas por Vector, Servicios de Salud,Veracruz, México.

(2) División Técnica de Información Estadística en Salud, Dirección de Prestaciones Médicas, Instituto Mexicano del Seguro Social (IMSS), México.

Fecha de recibido: 5 de junio de 2002 - Fecha de aprobado: 26 de septiembre de 2002

Solicitud de sobretiros: D r. Héctor Gómez Dantés. División Técnica de Información Estadística en Salud, D irección de Prestaciones Médicas, IMSS Correo electrónico:hdantes@ hotmail.com 
nantes sociales y ambientales de la transmisión. Este enfoque de riesgo puede mejorar el control y tener un impacto significativo en la prevención de la transmisión en las zonas de mayor riesgo. El texto completo en inglés de este artículo está disponible en: http://www.insp.mx/salud/index.html

Palabras clave: dengue; factores de riesgo; ecología; estratificación; México
Key words: dengue; risk factors; ecology; stratified; Mexico
A finales del siglo XX el dengue en las Américas emergió como uno de los problemas de salud pública de mayor relevancia, debido a la rápida expansión de la infestación del vector en la Región. Después de la campaña de erradicación del Aedes aegypti en la década de los 60, la reintroducción y reinfestación del vector se vieron favorecidas por el proceso de urbanización, la migración del campo a las ciudades, la carencia de servicios públicos en los crecientes centros urbanos y las deficiencias operativas de los programas de vigilancia y control de vectores en la Región. A partir de la epidemia de dengue hemorrágico $(\mathrm{DH})$ en Cuba en 1981, cada vez son más los países que reportan casos de DH en la Región. ${ }^{1}$

En las Américas la incidencia del dengue clásico ha ido en aumento a partir de la década de los 70 con ciclos epidémicos regulados por la introducción y circulación de los cuatros serotipos responsables. ${ }^{2}$ La forma severa de la infección, el dengue hemorrágico (FHD), es considerada como una de las enfermedades emergentes más importantes en el continente debido a la amplia distribución del vector, la naturaleza urbana de su transmisión, la letalidad y la carencia de tratamientos eficaces y vacunas que prevengan su incidencia. De 1980 a 1998 se reportaron 302330 casos de FHD en 24 países de las Américas, siendo Colombia el país con el mayor número de casos reportados (80 310), seguido por Brasil (55 150), Venezuela (54 514), Puerto Rico (41 942), Nicaragua (36 257) y Cuba (10517)., En México la tendencia del dengue clásico a partir de 1980 pareciera ser descendente aun cuando en 1993 se presentó un ligero incremento, situación que con seguridad respondió al mejoramiento de los sistemas de vigilancia. Fue en 1995 cuando se presentó la primera epidemia de fiebre hemorrágica de dengue, con 539 casos. $^{5,6}$

A pesar de la emergencia de este problema sanitario, la instrumentación de medidas de control no ha sido tan efectiva debido a que las campañas de descacharrización, la aplicación de larvicidas en los múltiples y numerosos criaderos, así como la aplicación de insecticidas, no han tenido la continuidad ni las coberturas necesarias para abatir las densidades del Aedes aegypti en las zonas urbanas, suburbanas y rurales donde se encuentra establecido el vector. Hoy todavía existe incertidumbre sobre la eficacia de las medidas de control emergente, como la aplicación a ultra bajo volumen (ULV, por sus siglas en inglés) de malatión, para contener la transmisión de dengue en caso de una epidemia. ${ }^{7}$ Por otro lado, la organización vertical de los programas de control carece de un enfoque de riesgo que estratifique las zonas de transmisión de acuerdo con las condiciones ecológicas y sociodemográficas de las regiones donde se transmite esta infección.

Las experiencias en las Américas, en particular la de la epidemia de dengue en Santiago de Cuba, ${ }^{8}$ han demostrado que aun en condiciones óptimas de control de las densidades vectoriales, la transmisión de los virus dengue puede establecerse de manera epidémica si no se mantiene una vigilancia epidemiológica estricta y permanente.

Frente a la emergencia del dengue en la Región y a la carencia de recursos humanos y materiales de los programas de control, son pocos los estudios que analizan las características ecológicas, sociales, demográficas y epidemiológicas que influyen sobre la dinámica de transmisión del mismo, para tratar de identificar las determinantes de la transmisión, y con ello orientar las estrategias de control de manera más selectiva y sustentadas en criterios de riesgo epidemiológico. En este estudio se describen las determinantes de la transmisión de dengue en las localidades del estado de Veracruz, México, se identifican la concentración y mantenimiento de la transmisión en cierto tipo de localidades y se propone la estratificación de riesgo de transmisión de acuerdo con las variables demográfi- 
cas (densidad de población), ecológicas (altitud sobre el nivel del mar) y sociales (marginación y dotación de servicios públicos).

\section{Material y métodos}

El diseño elegido para elaborar la estratificación de riesgo se basó en el abordaje ecológico. Para la conformación de la base de datos se empleó la información recabada en el formato de registro de las localidades que fueron positivas a dengue, generada por el programa de Prevención y Control de Enfermedades Transmitidas por Vector de los Servicios de Salud de Veracruz. * La información recogida en dicha base de datos incluye el número de casos de dengue que se presentaron durante el periodo 1995 a 1998 en las 1249 localidades ubicadas en los 174 municipios que conforman las 11 Jurisdicciones Sanitarias del estado de Veracruz.

A cada localidad se le agregaron los parámetros geográficos de altitud sobre el nivel del mar, latitud y longitud. La altitud se categorizó en tres niveles: por debajo de los 600 metros sobre el nivel del mar ( $\mathrm{msnm}$ ), de 600 a 1200 metros snm y por arriba de los 1200 metros snm. Las variables demográficas fueron el número total de habitantes, desagregados por sexo. Con esta información se construyó la variable de hacinamiento, estimando el número de habitantes por vivienda. Las localidades se clasificaron, con hacinamiento, si tenían en promedio cinco o más habitantes por vivienda. Los parámetros sociales utilizados fueron el número total de viviendas por localidad, número de viviendas ocupadas y el número de viviendas con dotación de servicios públicos: agua entubada, drenaje y electricidad. La dotación de servicios en cada localidad se analizó de acuerdo con el porcentaje de viviendas que contaban con cada uno de los servicios. Las categorías utilizadas fueron $<50 \%$, entre 50 y $75 \%$ y $>$ $75 \%$. Dicha información se obtuvo del Censo General de Población (1995). Cada localidad se clasificó respecto al grado de marginación, de acuerdo con el índice de marginación de localidades del Consejo Nacional de Población (CONAPO).

Las localidades también se identificaron de acuerdo con el número de años que reportaron dengue en el periodo estudiado, y se clasificaron como localidades repetidoras de dengue sólo a aquellas que reportaron

\footnotetext{
* Programa de Emergencias Epidemiológicas de Dengue y Paludismo. Servicios de Salud de Veracruz, 1999. Documento interno.
}

dengue en los cuatro años consecutivos, sin tomar en cuenta el número de casos.

Para fines del análisis, el estado se regionalizó en tres grandes áreas geográficas: la región norte, formada por las Jurisdicciones Sanitarias No. 1, de Pánuco, No. 2, de Tuxpan, No. 3, de Poza Rica y No. 4, de Martínez de la Torre. La región centro está conformada por las jurisdicciones No. 5, de Xalapa, No. 6, de Córdoba y No. 7, de Orizaba. Por último, la región sur está constituida por las Jurisdicciones No. 8, de Veracruz, No. 9 de Cosamaloapan, No. 10, de San Andrés Tuxtla y No. 11 de Coatzacoalcos.

El análisis estadístico bivariado se realizó tomando como variable dependiente el número de casos y la tasa de dengue categorizada en tres niveles. Se establecieron tres niveles de intensidad de transmisión: baja, cuando la tasa de incidencia fue menor a 5 por 100000 habitantes; media, si la tasa era de 5 a 10 por 100 000, y alta, si la tasa era superior a 10 por 100000 habitantes. Se usó el paquete estadístico de STATA 6.0 para Windows y se utilizó la prueba de ji cuadrada de Pearson para probar las diferencias entre las variables estudiadas.

\section{Resultados}

Veracruz es un estado endémico y ya tiene documentada la circulación de los cuatro serotipos. La circulación del serotipo DEN-3 se detectó en 1995 y desde entonces se incrementó la combinación de serotipos circulantes en la entidad. La tasa de dengue a escala estatal aumentó de 37.7 por 100000 habitantes, en 1994, a 112.7 en 1997. Durante 1997 se notificaron 10979 casos, incluyendo 192 hemorrágicos. Las Jurisdicciones Sanitarias más afectadas fueron Cosamaloapan (424 x 100 000), San Andrés (360.1), Coatzacoalcos (295.9) y Veracruz (216.5). En 1998, el número de casos disminuyó $77.4 \%$ con relación a 1997, y las Jurisdicciones más afectadas fueron Pánuco (108.2), Poza Rica (48), Tuxpan (44.2) y Cosamaloapan (43.2 por 100000 habitantes). En 1999 se registraron 2358 casos. Por lo que respecta al dengue hemorrágico, durante 1994 se presentaron cinco casos aislados. En 1995, se reportaron 77, con nueve defunciones. Durante 1996 esta forma clínica alcanzó la cifra más alta, con 512 casos y 17 defunciones; siendo la Jurisdicción de Veracruz la que más casos y defunciones acumuló ( 239 y 6, respectivamente). A partir de 1997 el dengue hemorrágico disminuyó $62 \%$ con relación al año anterior y sólo se registraron siete defunciones. En 1998, los casos de dengue hemorrágico fueron 44 , con tres defunciones.

La incidencia de dengue en el estado muestra diferencias regionales que distinguen variaciones tempo- 
rales y en magnitud de la transmisión. La transmisión de dengue en los últimos años demuestra que la región sur presenta las tasas más elevadas con un pico importante durante 1997 vinculado con la introducción del DEN-3. La región norte se mantiene con una tasa entre 50 y 100 casos por 100000 habitantes mientras que en la región centro existe un descenso importante durante los últimos años de los noventa con tasas inferiores a los 50 casos por 100000 habitantes. Como se verá más adelante, estas diferencias regionales responden a las características demográficas, ecológicas y sociales imperantes en las tres regiones.

El estado de Veracruz cuenta con un total 7450 localidades de las cuales sólo 1249 (17\%) reportaron dengue durante el periodo de estudio. $\mathrm{Al}$ interior de las Jurisdicciones dicha proporción varió de $6 \%$ de las localidades con dengue en la jurisdicción de Orizaba hasta $28 \%$ de las localidades con reporte de dengue en San Andrés Tuxtla. La mayor proporción de localidades con transmisión de dengue se presentó en la región sur. De los poco más de diez millones de habitantes en el estado, sólo 36\% radica en localidades con transmisión de dengue, es decir, la población en riesgo radica en sólo 17\% de las localidades del estado. De la misma manera, esta distribución varió entre cada Jursidicción, llegando a ser hasta de $44 \%$ en la de Coatzacoalcos (cuadro I).

De 1995 a 1998 se reportaron un total de 26423 casos de dengue en Veracruz. La región sur concentró $59 \%$ de los casos, seguida por la región norte, con $28 \%$. La tasa estatal fue de 716 por 100000 habitantes y presentó importantes variaciones entre las Jurisdicciones. Cosamaloapan, Veracruz y Poza Rica se distinguieron como las Jurisdicciones con las tasas más elevadas, (cuadro I).

En el ámbito estatal, las tasas de dengue se relacionaron significativamente con las variables sociales y demográficas (cuadro II). Aunque el dengue se considera un problema eminentemente urbano, el estado de Veracruz demuestra que también se transmite en las áreas rurales, ya que $85 \%$ de las localidades que reportaron dengue tuvieron menos de 2500 habitantes. Esto, a su vez, se ve reflejado en el grado de marginación de las localidades con dengue, ya que 63\% estuvieron clasificadas como de alta marginación.

El parámetro de altitud juega un papel importante en la dinámica de transmisión del dengue al observar que $87 \%$ de las localidades con dengue en el estado se ubicaron en el rango por debajo de los $600 \mathrm{msnm}$ y $59 \%$ estaban en el rango por debajo de los $100 \mathrm{msnm}$. Por sus condiciones geográficas, las Jurisdicciones Sanitarias del centro son las que tienen el mayor porcentaje de sus localidades afectadas por arriba de los 600 msnm, mientras que las con las tasas de dengue más elevadas tienen un número mayor de localidades ubicadas por debajo del rango de los $600 \mathrm{msnm}$. Esta diferencia fue significativa entre los diferentes niveles de transmisión, ya que la proporción de localidades con dengue por debajo de los $100 \mathrm{msnm}$ aumenta cuando la tasa pasa de 5 a 10 o más por cada 100000 habitantes (cuadro II).

La dotación de servicios públicos, entre las localidades que reportaron dengue y las que no registraron transmisión, nos señala que aquellas con dengue contaban, en promedio, con una mayor proporción de viviendas con agua potable, drenaje y electricidad. Aunque $85 \%$ de las localidades que reportaron dengue tenían menos de 2500 habitantes, la dotación de servicios públicos nos indica un grado importante de urbanización en las localidades con transmisión. Respecto a la dotación de agua potable y electricidad en las localidades con una baja tasa de transmisión $(<5 \mathrm{x}$ 100000 ), predominaron las localidades con más de $75 \%$ de las viviendas con este servicio. Sólo en $50 \%$ de las localidades con tasas medias y altas de transmisión, $75 \%$ o más de las viviendas contaban con estos servicios. Respecto al drenaje se observó que en $70 \%$ de las localidades con tasas medias y altas de transmisión, menos de 50\% de sus viviendas contaban con drenaje. Esto quiere decir que las tasas de dengue son más altas en aquellas localidades con una baja cobertura de los servicios públicos en las viviendas ya que la tasa se incrementa en la medida en la que disminuye la cobertura de los servicios.

El impacto que ejercen dichas variables sobre las diferencias regionales se expresan en su distribución entre y al interior de las jurisdicciones. La amplia dispersión de la transmisión de dengue se observa al constatar que más de $80 \%$ de las localidades con dengue tienen menos de 2500 habitantes. En las jurisdicciones del Centro, Orizaba y Córdoba, la transmisión en las áreas rurales es un poco menor. Son también las jurisdicciones del Centro, junto con Veracruz en el sur, las que presentan dengue en localidades menos marginadas y con mayor dotación de servicios que en el resto del estado.

El panorama descrito sólo alcanza a reseñar las características más generales de la epidemiología del dengue en el estado de Veracruz, ya que se adjudica el mismo peso a las localidades que reportan dengue en un año que a las que reportan dengue durante cada año del periodo en estudio. De igual forma, este análisis preliminar le otorga la misma importancia a una localidad que reporta un caso que a aquella que reporta 1000. Es por ello que se seleccionaron las localidades que reportaron dengue durante los cuatro años 


\section{Cuadro I}

\section{Dotación de servicios públicos en localidades con y sin dengue en Veracruz, México, 1995-1998}

$\begin{array}{cccccccc}\text { Región } & \text { Jurisdicción } & \text { Viviendas (\%) } & \text { Total de } & \text { Localidades } & \text { Población Población No. de } & \text { Tasa } x \\ \text { geográfica } & \text { sanitaria } & \text { Agua } & \text { electricidad } & \text { Drenaje localidades con dengue (\%) total en riesgo (\%) } & \text { casos } & 100000\end{array}$

\begin{tabular}{|c|c|c|c|c|c|c|c|c|c|c|}
\hline \multirow[t]{2}{*}{ Norte } & \multicolumn{10}{|l|}{ 1. Pánuco } \\
\hline & Sin dengue & 56 & 21 & 12 & 735 & & 396286 & & & \\
\hline & Con dengue & 83 & 53 & 43 & 104 & 12 & 180529 & 31 & 1302 & 721 \\
\hline \multicolumn{11}{|c|}{ 2. Tuxpan } \\
\hline & Sin dengue & 78 & 35 & 34 & 490 & & 396475 & & & \\
\hline & Con dengue & 88 & 60 & 56 & 106 & 18 & 173915 & 30 & 709 & 408 \\
\hline \multicolumn{11}{|c|}{ 3. Poza Rica } \\
\hline & Sin dengue & 64 & 13 & 14 & 729 & & 679245 & & & \\
\hline & Con dengue & 86 & 61 & 72 & 125 & 15 & 381364 & 36 & 4669 & 1224 \\
\hline \multicolumn{11}{|c|}{ 4. Martínez de la Torre } \\
\hline & Sin dengue & 76 & 59 & 45 & 522 & & 398669 & & & \\
\hline & Con dengue & 92 & 63 & 78 & 93 & 15 & 199780 & 33 & 695 & 348 \\
\hline
\end{tabular}

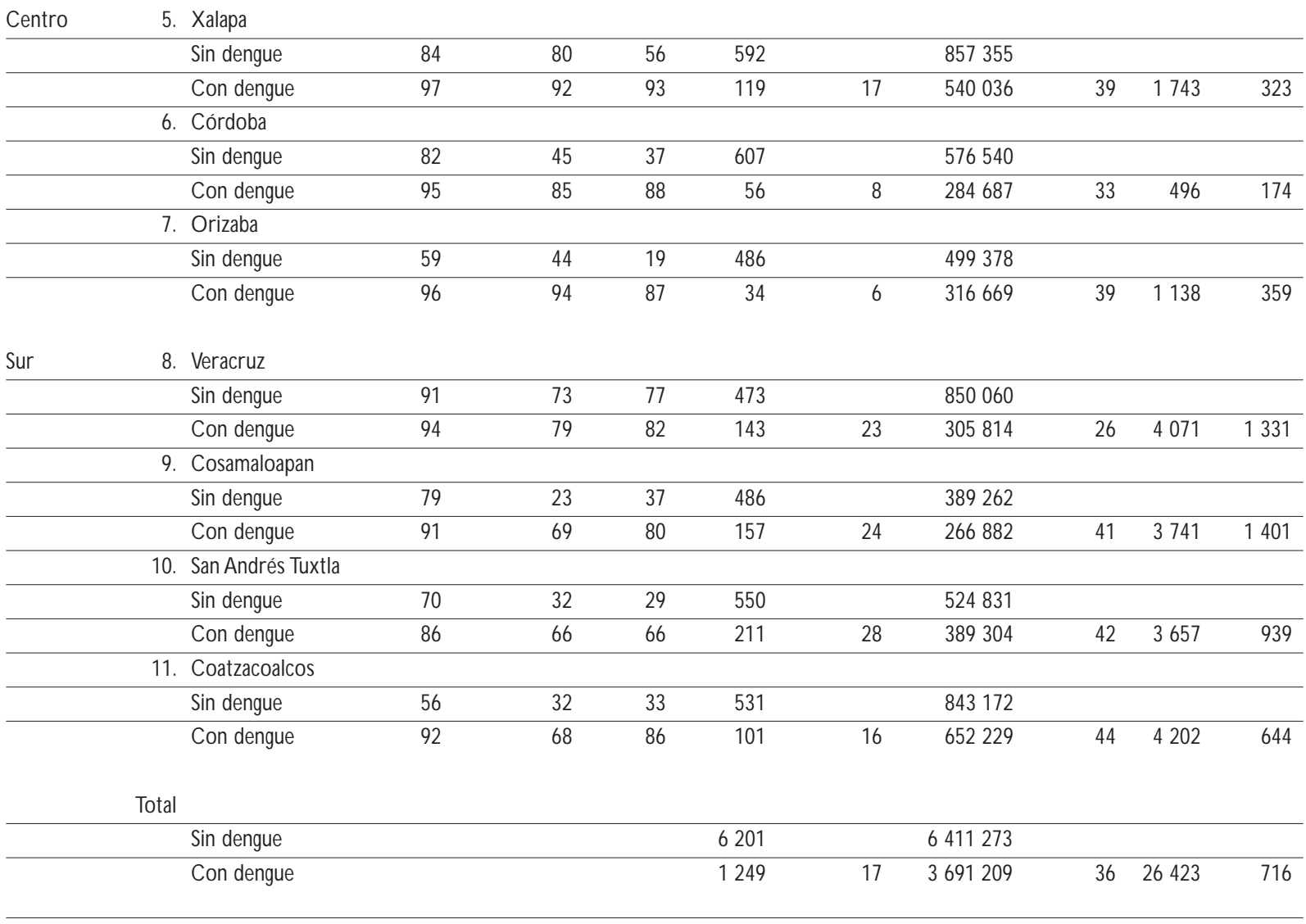

del estudio, y se les comparó con las que reportaron dengue en una, dos o tres ocasiones.

La información que arroja dicha estratificación se presenta en el cuadro III. Lo más relevante es que de las 1249 localidades identificadas con transmisión de dengue sólo $79(6 \%)$ fueron localidades repetidoras y en ellas se encontraba $57 \%$ de la población en riesgo salud pública de méxico / vol.45, no.1, enero-febrero de 2003 y se registraron $70 \%$ de los 26423 casos reportados en el estado para ese periodo. En algunos casos, la población en las localidades repetidoras rebasaba $70 \%$ del total de la población y $80 \%$ del total de casos en la Jurisdicción; por ejemplo, en Poza Rica, Veracruz y Coatzacoalcos. Esto quiere decir que enfocando los esfuerzos en un pequeño universo de localidades en la Jurisdic- 
Cuadro II

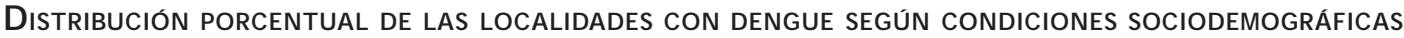 por Jurisdicción Sanitaria.Veracruz, México, 1995-1998}

Jurisdicciones Norte Centro Sur

No. de localidades Pánuco Tuxpan Poza Rica Martínez de la Torre Xalapa Córdoba Orizaba Veracruz Cosamaloapan San AndrésTuxtla Coatzacoalcos $(n=104)$ $(n=106) \quad(n=125) \quad(n=93)$ $(n=119)$

$(n=56) \quad(n=34) \quad(n=143) \quad(n=157) \quad(n=211)$ $(n=101)$

Variable

Población*

\begin{tabular}{rrrrrrrrrrrr}
\hline$<2500$ & 87 & 88 & 90 & 83 & 84 & 71 & 41 & $88^{\ddagger}$ & 88 & 89 & 79 \\
\hline$<10000$ & 7 & 9 & 5 & 13 & 13 & 18 & 44 & 9 & 8 & 7 & 13 \\
\hline$>10000$ & 6 & 3 & 5 & 4 & 3 & 11 & 15 & 3 & 4 & 4 & 8
\end{tabular}

Hacinamiento*

\begin{tabular}{llllllllllll}
\hline$<5$ & 50 & 54 & 30 & 78 & 68 & 41 & 47 & $90^{\ddagger}$ & 77 & $45^{\ddagger}$ & 49 \\
\hline$>5$ & 50 & 46 & 70 & 22 & 32 & 59 & 53 & 10 & 23 & 55 & 51
\end{tabular}

Marginación*

\begin{tabular}{llrrrrrrrrrr}
\hline Baja & 10 & 9 & 6 & 31 & 56 & 20 & 32 & $33^{\ddagger}$ & 15 & $15^{\ddagger}$ & 10 \\
\hline Media & 17 & 10 & 6 & 25 & 23 & 27 & 18 & 28 & 21 & 21 & 11 \\
\hline Alta & 73 & 81 & 88 & 44 & 21 & 53 & 50 & 39 & 64 & 64 & 79
\end{tabular}

Altitud*

\begin{tabular}{lrrrrrrrrrrr}
\hline$<100 \mathrm{msnm}$ & 53 & 65 & 43 & 62 & $13^{\ddagger}$ & 6 & 0 & 87 & 90 & $62^{\ddagger}$ & 87 \\
\hline $101-600 \mathrm{msnm}$ & 45 & 35 & 54 & 27 & 23 & 46 & 0 & 12 & 10 & 37 & 13 \\
\hline$>600 \mathrm{msnm}$ & 2 & 0 & 3 & 11 & 64 & 48 & 100 & 1 & 0 & 1 & 0
\end{tabular}

Agua*

\begin{tabular}{lrrrrrrrrrrr}
\hline$<50 \%$ & 59 & 49 & 64 & 13 & $8^{\ddagger}$ & 27 & $3^{\ddagger}$ & $30^{\ddagger}$ & 41 & 45 & 55 \\
\hline $75 \%$ & 5 & 6 & 6 & 3 & 0 & 2 & 3 & 4 & 6 & 4 & 5 \\
\hline$>75 \%$ & 36 & 45 & 30 & 84 & 92 & 71 & 94 & 66 & 53 & 51 & 40
\end{tabular}

Electricidad*

\begin{tabular}{lrrrrrrrrrrr}
\hline$<50 \%$ & 43 & $28^{\ddagger}$ & 34 & 23 & $19^{\ddagger}$ & $21^{\ddagger}$ & $6^{\ddagger}$ & $30^{\ddagger}$ & 37 & 34 & $49^{\ddagger}$ \\
\hline $75 \%$ & 15 & 15 & 7 & 12 & 13 & 9 & 6 & 11 & 13 & 11 & 8 \\
\hline$>75 \%$ & 42 & 57 & 59 & 65 & 68 & 70 & 88 & 59 & 50 & 55 & 43
\end{tabular}

Drenaje*

\begin{tabular}{lrrrrrrrrrrr}
\hline$<50 \%$ & $84^{\ddagger}$ & 75 & 79 & 44 & $32^{\ddagger}$ & 48 & 24 & $50^{\ddagger}$ & 61 & 70 & $60^{\ddagger}$ \\
\hline $75 \%$ & 1 & 5 & 5 & 6 & 11 & 6 & 3 & 7 & 8 & 7 & 4 \\
\hline$>75 \%$ & 15 & 20 & 16 & 50 & 57 & 46 & 73 & 43 & 31 & 23 & 36
\end{tabular}

Tasa*

\begin{tabular}{lrrrrrrrrrrr}
\hline $5 \times 100000$ & 58 & 67 & 56 & 100 & 52 & 89 & 70 & 57 & 31 & 41 & 51 \\
\hline $5-10 \times 100000$ & 24 & 17 & 24 & 0 & 23 & 7 & 12 & 23 & 27 & 22 & 31 \\
\hline$>10 \times 100000$ & 18 & 16 & 20 & 0 & 25 & 4 & 18 & 20 & 42 & 37 & 18
\end{tabular}

* $p<0.001$ señala que las diferencias entre cada Jurisdicción son significativas

₹ $p<0.05$ señala que la tasa de dengue de cada Jurisdicción fue diferente al interior de los estratos de cada variable

msnm: metros sobre el nivel del mar 


\section{Cuadro III \\ Distribución de los CASOS DE DENGUE EN LAS LOCALIDADES REPETIDORAS en Veracruz, México, 1995-1998}

\begin{tabular}{|c|c|c|c|c|c|c|c|c|c|}
\hline $\begin{array}{l}\text { Región } \\
\text { geográfica }\end{array}$ & $\begin{array}{l}\text { Jurisdicción } \\
\text { sanitaria }\end{array}$ & $\begin{array}{l}\text { Localidades } \\
\text { con dengue }\end{array}$ & $\begin{array}{l}\text { Localidades } \\
\text { repetidoras } \\
\text { n (\%) }\end{array}$ & $\begin{array}{l}\text { Población total } \\
\text { en localidades } \\
\text { con dengue* }\end{array}$ & $\begin{array}{c}\text { Población total } \\
\text { en localidades } \\
\text { repetidoras n (\%) }\end{array}$ & $\begin{array}{c}\text { Casos en } \\
\text { localidades } \\
\text { con dengue* }\end{array}$ & $\begin{array}{l}\text { Casos de dengue } \\
\text { en localidades } \\
\text { repetidoras } n(\%)\end{array}$ & $\begin{array}{l}\text { Tasa en localidades } \\
\text { con dengue } \\
\text { X } 100000^{*}\end{array}$ & $\begin{array}{c}\text { Tasa en localidades } \\
\text { repetidoras } \\
\times 100000^{\ddagger}\end{array}$ \\
\hline \multirow[t]{4}{*}{ Norte } & 1 Pánuco & 104 & $5(5)$ & 103676 & $76853(43)$ & 696 & $606(46)$ & 671 & 788 \\
\hline & 2 Tuxpan & 106 & $5(5)$ & 101176 & $72739(42)$ & 382 & $327(46)$ & 377 & 449 \\
\hline & 3 Poza Rica & 125 & $9(7)$ & 102637 & $278727(73)$ & 558 & $4111(88)$ & 543 & 1475 \\
\hline & 4 Martínez de la Torre & 93 & $1(1)$ & 155921 & $43859(22)$ & 572 & $123(18)$ & 367 & 280 \\
\hline \multirow[t]{3}{*}{ Centro } & 5 X alapa & 119 & $9(8)$ & 151457 & $388579(72)$ & 974 & 769 (44) & 643 & 198 \\
\hline & 6 Córdoba & 56 & $2(4)$ & 147545 & $137142(48)$ & 180 & $316(64)$ & 122 & 230 \\
\hline & 70 rizaba & 34 & $5(15)$ & 224848 & $91821(29)$ & 406 & $732(64)$ & 180 & 797 \\
\hline \multirow[t]{4}{*}{ Sur } & 8 Veracruz & 143 & $9(6)$ & 122715 & $183099(60)$ & 516 & 3555 (87) & 420 & 1942 \\
\hline & 9 Cosamaloapan & 157 & $13(8)$ & 111256 & $155626(58)$ & 1336 & $2405(64)$ & 1200 & 1545 \\
\hline & 10 San Andrés Tuxtla & 211 & $15(7)$ & 187049 & $202255(52)$ & 1641 & $2016(55)$ & 877 & 997 \\
\hline & 11 Coatzacoalcos & 101 & $6(6)$ & 168783 & 483446 (74) & 746 & $3456(82)$ & 442 & 715 \\
\hline Total & & 1249 & $79(6)$ & 1577063 & 2114146 (57) & 8007 & $18416(70)$ & 742 & 871 \\
\hline
\end{tabular}

ción se puede atacar la mayor parte del problema en la región. En términos de tasas por 100000 habitantes, la de las localidades repetidoras es muy superior que en aquellas donde reportaron dengue de manera ocasional. Además, las tasas en las Jurisdicciones de Córdoba y Orizaba son las más bajas respecto a las localidades con dengue ocasional, y las de Jalapa y Córdoba presentan las tasas más bajas en las localidades repetidoras. Esto apoya la presencia de determinantes ecológicas y sociales de la transmisión en el estado al constatar que aun en condiciones de alta transmisión , la región centro es la que presenta menores tasas de dengue.

A diferencia del total de las localidades con dengue, las localidades repetidoras son eminentemente más urbanas, de mayor tamaño, con menores condiciones de marginación y con una dotación más amplia de agua potable, drenaje y electricidad (cuadro IV). En cuanto a las tasas de dengue, la región centro manifestó las menores (<5 x 100 000) en la mayoría de sus localidades mientras que la región sur tuvo una mayor proporción de sus localidades con tasas superiores a los 5 casos por cada 100000 habitantes. Una secuencia lógica de estos resultados es que no sólo existen diferencias regionales en cuanto al número de lo- calidades y localidades repetidoras sino que, además, el enfoque hacia las Jurisdicciones de mayor riesgo impactaría en mayor grado la incidencia del dengue en todo el estado.

La diferencia de riesgos entre las localidades repetidoras y las localidades con dengue ocasional se observa claramente en el Cuadro V. Aquí se demuestra que el número promedio de casos por localidad cuando se reporta dengue en uno, dos, tres o cuatro años del periodo es sustantivamente diferente. Por ejemplo, en la Jurisdicción de Pánuco se reportan tres casos de dengue al año en promedio en las localidades que sólo reportaron dengue en un solo año durante el periodo de estudio, pero aumenta a 121 en promedio por año cuando se trata de localidades que reportaron transmisión de dengue durante los cuatro años que abarca el periodo estudiado.

Más importante aún es el hallazgo de un umbral de población indispensable para que el dengue se mantenga presente en una localidad durante el periodo de estudio. Las localidades que reportaron dengue en una sola ocasión tienen menos de 1000 habitantes en promedio. y las localidades que reportaron en dos ocasiones tienen menos de 2500 habitantes en promedio. Estas localidades son de naturaleza rural, con su población 


\section{Cuadro IV}

Distribución PORCENTUAL de LAS LOCALIDADES REPETIDORAS DE DENGUE SEGÚN CONDICIONES SOCIOdemográficas comparadas con las no Repetidoras. VeracruZ, MéXico, 1995-1998

\begin{tabular}{|c|c|c|c|c|c|c|c|c|c|c|c|}
\hline \multirow[b]{2}{*}{$\begin{array}{l}\text { Jurisdicciones/localidades } \\
\text { con dengue/localidades }\end{array}$} & \multicolumn{4}{|c|}{ Norte } & \multicolumn{3}{|c|}{ Centro } & \multicolumn{4}{|c|}{ Sur } \\
\hline & $\begin{array}{l}\text { Pánuco } \\
\text { ( } n=104)\end{array}$ & $\begin{array}{l}\text { Tuxpan } \\
\text { (n=106) }\end{array}$ & $\begin{array}{c}\text { Poza Rica } \\
(n=125)\end{array}$ & $\begin{array}{l}\text { Martínez de la Torre } \\
\qquad(\mathrm{n}=93)\end{array}$ & $\begin{array}{l}\text { Xalapa } \\
(n=119)\end{array}$ & $\begin{array}{l}\text { Córdoba } \\
(n=56)\end{array}$ & $\begin{array}{r}\text { Orizaba } \\
(n=34)\end{array}$ & $\begin{array}{l}\text { Veracruz } \\
(n=143)\end{array}$ & $\begin{array}{l}\text { Cosamaloapan } \\
(n=157)\end{array}$ & $\begin{array}{l}\text { San Andrés Tuxtla } \\
(n=211)\end{array}$ & $\begin{array}{l}\text { Coatzacoalcos } \\
(n=101)\end{array}$ \\
\hline & $5(5 \%)$ & $5(5 \%)$ & $9(7 \%)$ & $1(1 \%)$ & $9(8 \%)$ & $2(4 \%)$ & $5(15 \%)$ & $9(6 \%)$ & $13(8 \%)$ & $15(7 \%)$ & $6(6 \%)$ \\
\hline
\end{tabular}

Variables:

Población

\begin{tabular}{rrrrrrrrrrrr}
\hline$<2500$ & $0^{\ddagger}$ & $20^{\ddagger}$ & $11^{\ddagger}$ & $0^{\ddagger}$ & $33^{\ddagger}$ & $0^{5}$ & $0^{\ddagger}$ & $22^{\ddagger}$ & $31^{\ddagger}$ & $20^{\ddagger}$ & $0^{\ddagger}$ \\
\hline$<10000$ & 40 & 20 & 22 & 0 & 44 & 50 & 20 & 44 & 31 & 40 & 0 \\
\hline$>10000$ & 60 & 60 & 67 & 100 & 22 & 50 & 80 & 33 & 38 & 40 & 100
\end{tabular}

Hacinamiento

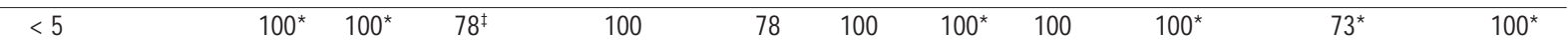

Marginación

\begin{tabular}{lrrrrrrrrrrr}
\hline Baja & $100^{\ddagger}$ & $80^{\ddagger}$ & $56^{\ddagger}$ & 100 & 67 & $100^{*}$ & $80^{*}$ & $100^{\ddagger}$ & $61^{\ddagger}$ & $60^{\ddagger}$ & $100^{\ddagger}$ \\
\hline Alta & 0 & 0 & 33 & 0 & 11 & 0 & 20 & 0 & 23 & 20 & 0 \\
\hline Media & 0 & 20 & 11 & 0 & 22 & 0 & 0 & 0 & 16 & 20 & 0
\end{tabular}

Agua
\begin{tabular}{lrrrrrrrrrrr}
\hline$<50 \%$ & $0 *$ & 20 & $11^{\ddagger}$ & 0 & 11 & 0 & 0 & 0 & 15 & $7^{*}$ & $0 *$ \\
\hline $75 \%$ & 0 & 0 & 0 & 0 & - & 0 & 0 & 0 & 8 & 0 & 0 \\
\hline$>75 \%$ & 100 & 80 & 89 & 100 & 89 & 100 & 100 & 100 & 77 & 93 & 100
\end{tabular}

Electricidad

\begin{tabular}{lrrrrrrrrrrr}
\hline$<50 \%$ & $0 *$ & 0 & $0 *$ & 0 & 11 & 0 & 0 & $0 *$ & $8 *$ & $7^{*}$ & $0 *$ \\
\hline $75 \%$ & 0 & 0 & 0 & 0 & 11 & 0 & 0 & 0 & 0 & 0 & 0 \\
\hline$>75 \%$ & 100 & 100 & 100 & 100 & 78 & 100 & 100 & 100 & 92 & 93 & 100
\end{tabular}

Drenaje

\begin{tabular}{lrrrrrrrrrrr}
\hline$<50 \%$ & $0^{\ddagger}$ & $20^{*}$ & $11^{\ddagger}$ & 0 & 22 & 0 & 0 & $0^{*}$ & $8^{\ddagger}$ & $7^{\ddagger}$ & $0^{*}$ \\
\hline $75 \%$ & 0 & 0 & 0 & 0 & 0 & 0 & 0 & 11 & 0 & 7 & 0 \\
\hline$>75 \%$ & 100 & 80 & 89 & 100 & 78 & 100 & 100 & 89 & 92 & 86 & 100
\end{tabular}

Tasa

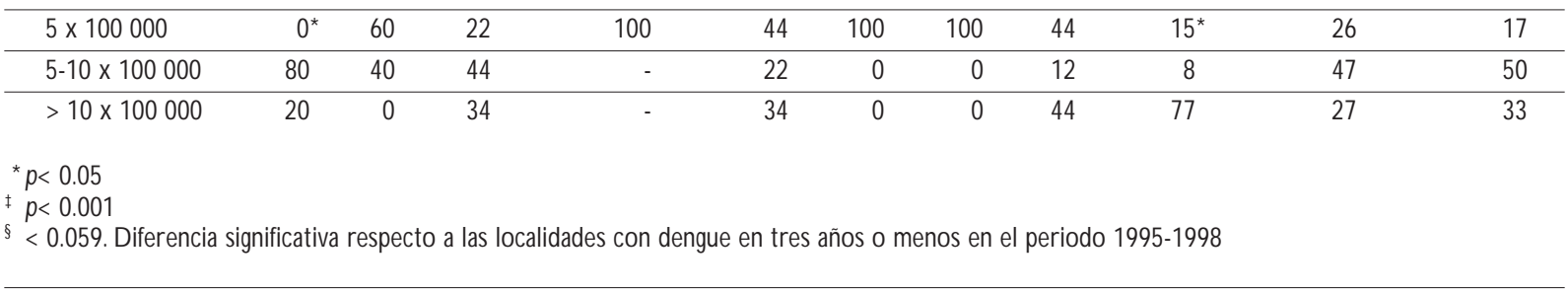

dispersa, mal comunicada y con una dotación pobre de servicios públicos. La transmisión de dengue en estas localidades es muy esporádica y ocasional, y seguramente es introducida a este tipo de comunidades por el contacto con otros centros urbanos. El dengue en estas localidades es dependiente de la transmisión en centros de mayor tamaño. Las que reportaron dengue en tres años están por debajo de los 10000 habitantes en promedio, y se podría decir que son localidades donde la transmisión sí logra mantenerse por el acúmulo y la introducción de susceptibles, debido a movimientos migratorios, comercio, empleo, etcétera. Las 


\section{Cuadro V \\ Promedio de casos y promedio de habitantes por localidad de acuerdo al número de años Con Reporte de CASOS de dengue.Veracruz, México, 1995-1998}

\begin{tabular}{|c|c|c|c|c|c|c|c|c|}
\hline \multirow[b]{3}{*}{ Jurisdicción sanitaria } & \multicolumn{8}{|c|}{ Número de años de reporte } \\
\hline & \multicolumn{2}{|r|}{1} & \multicolumn{2}{|r|}{2} & \multicolumn{2}{|l|}{ 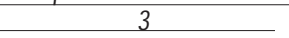 } & \multicolumn{2}{|r|}{4} \\
\hline & $\begin{array}{l}\text { Casos por } \\
\text { localidad }\end{array}$ & $\begin{array}{l}\text { Habitantes por } \\
\text { localidad }\end{array}$ & $\begin{array}{l}\text { Casos por } \\
\text { localidad }\end{array}$ & $\begin{array}{l}\text { Habitantes por } \\
\text { localidad }\end{array}$ & $\begin{array}{l}\text { Casos por } \\
\text { localidad }\end{array}$ & $\begin{array}{l}\text { Habitantes por } \\
\text { localidad }\end{array}$ & $\begin{array}{l}\text { Casos por } \\
\text { localidad }\end{array}$ & $\begin{array}{l}\text { Habitantes por } \\
\text { localidad }\end{array}$ \\
\hline 1 Pánuco & 3 & 714 & 3 & 1045 & 120 & 7828 & 121 & 15371 \\
\hline 2 Tuxpan & 2 & 647 & 5 & 1761 & 10 & 3616 & 65 & 14548 \\
\hline 3 Poza Rica & 2 & 764 & 6 & 1182 & 17 & 1058 & 457 & 30970 \\
\hline 4 Martínez de la Torre & 4 & 910 & 9 & 1556 & 22 & 10729 & 123 & 43859 \\
\hline 5 Xalapa & 2 & 989 & 13 & 2131 & 28 & 2300 & 85 & 43175 \\
\hline 6 Córdoba & 2 & 1793 & 5 & 3789 & 20 & 15722 & 158 & 68571 \\
\hline 70 rizaba & 5 & 2930 & 27 & 2483 & 11 & 3569 & 146 & 40012 \\
\hline 8 Veracruz & 1 & 577 & 6 & 1253 & 20 & 3342 & 395 & 20344 \\
\hline 9 Cosamaloapan & 3 & 511 & 4 & 338 & 30 & 3100 & 185 & 11971 \\
\hline 10 San Andrés Tuxtla & 3 & 525 & 6 & 819 & 28 & 2128 & 143 & 9752 \\
\hline 11 Coatzacoalcos & 3 & 983 & 14 & 3262 & 51 & 12635 & 576 & 80574 \\
\hline
\end{tabular}

localidades repetidoras son aquellas que tienen más de 30000 habitantes en promedio y son centros urbanos establecidos, cuentan con una intensa actividad económica, comercial, con continuos movimientos de población y están bien comunicadas y ubicadas en zonas geográficas que permiten el establecimiento, el mantenimiento y la proliferación del vector.

\section{Discusión}

Existe una amplia experiencia en la Región de las Américas que demuestra que el dengue no se transmite de manera homogénea, ni afecta por igual a las poblaciones que residen en las áreas donde se establece como problema de salud pública. ${ }^{9-13}$ Las similitudes adjudicadas a la epidemia de dengue y FHD en Asia y las Américas desaparecen cuando se analiza la dinámica de transmisión dentro de cada país, región, estado, municipio y localidad. ${ }^{14}$ El dengue no se transmite de manera igual en todo México pues hay estados que son endémicos y otros que todavía se resisten al establecimiento del vector y a la transmisión de la infección. ${ }^{15}$

Este trabajo confirma que el dengue sí se transmite en las áreas rurales y que dejó de ser un padecimiento de naturaleza urbana aunque, al parecer, la transmisión del dengue es ocasional, esporádica y de muy baja magnitud; ello implica que las condiciones necesarias para la proliferación del vector y la transmisión del virus ya se encuentran plenamente establecidas y diseminadas en las áreas rurales del estado. El hecho de que cerca de $80 \%$ de las localidades que reportaron dengue durante el periodo tengan menos de 2500 habitantes es indicativo de que las vías de comunicación y transporte ya facilitan la introducción de la infección a comunidades antes consideradas poco adecuadas para la transmisión. Esto nos habla de la enorme facilidad que tienen los virus dengue de penetrar en comunidades aisladas y afectar poblaciones dispersas. No deja de ser importante la carga provocada por el número de casos acumulados en las áreas rurales, pero el patrón que los distingue del resto de la epidemia del dengue en el estado es que son casos esporádicos y ocasionales. Así lo demuestra el número promedio de tres casos por localidad cuando se reporta en una sola ocasión, o de nueve casos en promedio por localidad, cuando se reporta en dos ocasiones durante el periodo.

Está bien reconocido el nivel de subregistro que existe de los casos de dengue aun en áreas donde existe un adecuado sistema de vigilancia. Es de esperarse que el nivel de subregistro en las áreas rurales dispersas, y con difícil acceso a los servicios de salud, sea aún mayor. Sin embargo, lo que aquí se destaca es que la naturaleza de dispersión y de mala comunicación de estas comunidades sí permite la introducción de la 
infección, pero no el establecimiento de las cadenas de transmisión indispensables para desarrollar epidemias importantes.

En cuanto a la variable hacinamiento, sólo podemos mencionar que se trata de las desventajas a las que se enfrenta uno cuando la variable exposición se adjudica al grupo y no al individuo. En este caso, las localidades fueron clasificadas con y sin hacinamiento, de acuerdo con el número total de individuos y de viviendas. El rol que juega el hacinamiento en la transmisión de dengue en cada localidad está determinado a escala microrregional y no tan general. ${ }^{16}$

Otro hallazgo importante que se desprende del análisis es que existe una clara tendencia del dengue a concentrarse en ciertas áreas urbanas, y que se identificaron algunas determinantes de la transmisión que permiten establecer una estratificación del riesgo de transmisión, que apoye los programas de control y vigilancia. Las localidades repetidoras de dengue son aquellas que tienen mayor población, menores condiciones de marginación y mayor dotación de servicios públicos. Es decir, son centros urbanos bien establecidos, con amplios canales de comunicación y de transporte, con una intensa actividad económica que favorece el desplazamiento de las poblaciones susceptibles. Su ubicación en regiones tropicales y subtropicales, por debajo de los $600 \mathrm{msnm}$, es indicativa de las condiciones ecológicas propicias para la proliferación de altas densidades vectoriales. En este trabajo también se demuestra que, inclusive al interior del estado de Veracruz, de las Jurisdicciones y las localidades existen diferencias en la dinámica de transmisión del dengue. El hecho de que $59 \%$ de los casos, $49 \%$ de las localidades afectadas y $44 \%$ de la población en riesgo se ubiquen en la región sur del estado nos habla de una concentración geográfica del dengue, que se intensifica cuando se observan las localidades repetidoras. Si 70\% de los casos en Veracruz se concentran en 79 localidades, la región sur contribuye con 43 localidades (54\%), con $43 \%$ del total de casos en el estado y $62 \%$ del total de casos en las localidades repetidoras. Estos hallazgos demandan la concentración de los esfuerzos en las localidades repetidoras y, en especial, el control de dengue en la región sur.

Las diferencias encontradas en cuanto al número promedio de casos reportados por localidad para el periodo de cuatro años presenta, además, un indicador adicional de riesgo, ya que se observa que las localidades repetidoras tienen un umbral poblacional mayor que las que sólo reportan dengue en una o dos ocasiones. Estos umbrales de población son determinantes para mantener la transmisión en un área y también señalan lo difícil que es establecer una cadena de transmisión en localidades con bajas densidades de población. La clasificación de localidades de mayor riesgo por su densidad poblacional es otro beneficio del proceso de estratificación.

La identificación de las localidades repetidoras va de la mano con lo manifestado por Barrera y colaboradores, en Venezuela, sobre los focos calientes, fríos y helados, aunque su propuesta se limita al área metropolitana de Maracay. ${ }^{17}$ En este estudio se identifican localidades que actúan como focos calientes a escala regional que seguramente influyen sobre la transmisión de dengue en los focos medianos y pequeños (fríos y helados). Es de esperarse que la transmisión en las localidades no repetidoras, de menor tamaño poblacional, y en condiciones de mayor marginación, sea altamente dependiente de lo que sucede en las localidades repetidoras de dengue. Es en este reducido número y tipo de localidades donde se concentra más de $50 \%$ de la población en riesgo y el número total de casos. Es por ello que se propone el control intensivo en estas localidades, para incidir de manera más eficiente sobre la transmisión de dengue. Un hecho adicional es que reduciendo la transmisión de dengue en estas localidades se incidiría también sobre el riesgo de dengue hemorrágico.

Ante la reducida capacidad operativa, y al limitado impacto de las intervenciones de los programas de control, es indispensable considerar estrategias que permitan un uso más adecuado de los limitados recursos con los que se cuenta en el ámbito local. En el estado de Veracruz, como en cualquier otra parte del país y la Región de las Américas y El Caribe, no se cuenta con el personal, los vehículos, el equipo y los insumos necesarios para atender el amplio abanico de localidades afectadas por el dengue. ${ }^{18}$ Es probable que no se disponga de recursos suficientes para, inclusive, atender la totalidad de las localidades repetidoras en el estado de manera intensiva y permanente. Sin embargo, este enfoque de riesgo y esta estrategia de estratificación permiten dirigir los escasos recursos hacia las zonas más problemáticas e intensificar los esfuerzos hacia las áreas más vulnerables dentro de cada localidad. Frente a la demanda de un control más efectivo deben proponerse estrategias más eficientes, y este trabajo contribuye con ese esfuerzo.

\section{Referencias}

1. Gubler DJ,Trent DW. Emergence of epidemic dengue/dengue hemorrhagic fever as a public health problem in the Americas. Infect Agents $D$ is 1994;2:383-393.

2. Gomez-Dantés H. El dengue en las Américas: un problema de salud regional. Salud Publica Mex 1991;33:347-355. 
3.W orld Health 0 rganization. Report on global surveillance of epidemicprone infectious diseases. Ginebra: W HO/CDS/C SR/ISR/, O rganización Mundial de la Salud, Ginebra, 2000.

4. Pinheiro F, Corber S, G lobal situation of dengue and dengue hemorrhagic fever, and its emergence in the Americas. World Health Stat Q 1997;50:161-169.

5. N arro-Robles J, Gómez-D antés H. El dengue en México: un problema prioritario de salud pública. Salud Publica Mex 1995;37supl:12-20.

6. Gómez-D antés H, Henry-Rodríguez M. El dengue y la fiebre hemorrágica del dengue: clínica y epidemiología.A sociación Mexicana de Infectología y Microbiología C línica 2000;B-5:1-40.

7. N ewton E, Reiter P.A model of the transmission of dengue fever with an evaluation of the impact of ultra-low volume (ULV) insecticide applications on dengue epidemics. Am J Trop Med Hyg, 1992;47:709-720.

8.Valdés L, G uzmán MG , Kourí G, Delgado J, C arbonell I, C abreraVM et al. Epidemiology of dengue and hemorrhagic dengue in Santiago, Cuba 1997. Rev Panam Salud Publica 1999;6(1):16-25.

9. Koopman JS, Prevots, DR, Vaca-Marín MA, Gómez-Dantés H, Zárate $A M L$, Longini MI et al. D eterminants and predictors of dengue infection in México.Am J Epidemiol 1991;133:1168-1178.

10. Gómez-D antés H, Koopman SJ,Addy CL, Zárate AML,Vaca-Marín MA, Longini MI et al. Dengue outbreaks in the Pacific Coast of Mexico. Int J Epidemiol 1988;17:178-186

11. Herrera-Basto E,Prevots D, Zárate ML, Silva L, Sepúlveda-A mor J. First report outbreak of clasical dengue fever at 1700 meters above sea-level in Guerrero State, Mexico.AM J Trop Med Hyg 1992;46(6):649-653.
12. Barrera R, N avarro JC, Mora J, D omínguez D, G onzález J. Public service deficiencies and Aedes aegypti breeding sites in Venezuela. Bull Pan Am Health 0 rgan 1995;29:193-205.

13. Paulino A, N atal D. G eographical distribution of dengue and socioeconomic factors in an urban locality in Southeastern Brazil. Rev Saude Publica 1998;32(3):232-236.

14. Aiken $S$, Frost D, Leigh $C$. D engue Hemorrhagic fever and rainfall in Peninsular Malaysia: Some suggested relationships. Soc Sci Med 1980;14D:307-316.

15. Gómez-Dantés H, Ramos BB, Tapia CR. El riesgo de transmisión del dengue: un espacio para la estratificación. Salud Publica Mex 1995;37 supl:88-97.

16. Morrison AC, G etis A, Santiago M, Rigau-Pérez J, Reiter P. Exploratory space-time analysis of reported dengue cases during an outbreak in Florida, Puerto Rico 1991-1992. Am J Trop Med Hyg 1998;58:287-298.

17. Barrera R, Delgado N , Jiménez M,Villalo bos I, Romero I. Estratificación de una ciudad hiperendémica en dengue hemorrágico. Rev Panam Salud Publica/ Pan Am J Public Health 2000;8(4):

18. 0 rganización Panamericana de la Salud. Dengue y dengue hemorrágico en las Américas: guía para su prevención y control. W ashington D.C.: O PS, 1994;Publicación Científica N 0. 548. 